\title{
Perceived Stress and Accompanying Low Urine pH Are in Relation to Bladder Pain Syndrome
}

\author{
(D) Ahmet Cihan1, (1) Esra Cihan2, (1) Bülent Çakmak² \\ ${ }^{1}$ Niğde Training and Research Hospital, Clinic of Urology, Niğde, Turkiye \\ 2Niğde Ömer Halisdemir University Faculty of Medicine, Department of Obstetrics and Gynecology, Niğde, Turkiye
}

What's known on the subject? and What does the study add?

Emotional stress has been demonstrated to be associated with symptom exacerbations in patients with bladder pain syndrome (BPS). Some clinical studies have also revealed that urinary alkalisation therapy improved symptoms in patients with BPS or hypersensitive bladder. This prospective case-control study evaluated the contributions and association of perceived stress accompanied with acidic urine with symptoms of BPS. Perceived stress is independent of urine $\mathrm{pH}$, as they each relate to BPS symptoms in a bidirectional manner. Perceived stress and urine $\mathrm{pH}$ should be evaluated in patients with BPS.

\begin{abstract}
Objective: The clinical relevance of low urine $\mathrm{pH}$ and perceived stress levels in patients with bladder pain syndrome (BPS) has not yet been clarified. In this study, we hypothesised that urine $\mathrm{pH}$ and perceived stress levels may differ in patients with BPS and that they may be related to each other. Materials and Methods: A prospective case-control study was conducted to test the hypothesis. Patients aged $>18$ years who were newly diagnosed with BPS were included in the patient group. The control group consisted of healthy volunteers aged $>18$ years. The 10-item Perceived Stress scale (PSS-10), spot urine pH measurements, Interstitial Cystitis Symptom index (ICSI), Interstitial Cystitis Problem index (ICPI), visual analogue scale for pain (VAS-Pain) and quality-of-life (OOL) scores were evaluated. Independent samples t-test and multivariate regression with path analysis were performed.

Results: The study evaluated 84 patients with BPS and 86 healthy participants. The mean spot urine pH, PSS-10, ICSI, ICPI, VAS-Pain and QOL scores were different between the patient group and control group. Spot urine $\mathrm{pH}$ level $[p=0.01$, odds ratio $(\mathrm{OR})=0.31]$ and $P S S-10$ scores $(p=0.01,0 R=1.1)$ remained significant predictors of BPS in the multivariate analysis. Lower urine $\mathrm{pH}$ and higher perceived stress levels were associated with worse ICSI, ICPI, VAS-Pain and OOL scores.

Conclusion: Acidic urine $\mathrm{pH}$ and high perceived stress levels are associated with the presence of BPS. Perceived stress is independent from low urine $\mathrm{pH}$, as they each relate to BPS symptoms in a bidirectional manner.
\end{abstract}

Keywords: Acidic urine, bladder pain, interstitial cystitis, perceived stress

\section{Introduction}

Since Alexander JC Skene coined the name "interstitial cystitis" in 1887, its definition, estimated prevalence and pathophysiological aspects investigated according to its subtypes have changed considerably over time (1). The phenotypic heterogeneity of the disorder and its associations restrains the physicians when treating patients with persisting symptoms of bladder pain syndrome (BPS). Promising results have been reported in recent studies on the therapeutic effects of urinary alkalisation therapy $(2,3)$. However, incidental findings in these studies such as improving urgency, frequency and pain with medical urine alkalisation barely reveal the aetiological relationships with urine $\mathrm{pH}$. This issue formed the basis for the first hypothesis of this study: Urine $\mathrm{pH}$ levels differ between patients with BPS and individuals without BPS. Low urine $\mathrm{pH}$ as a marker of renal acid load is also an under-investigated clinical finding. Metabolic acidosis, dietary acid load and renal

Correspondence: Ahmet Cihan MD, Niğde Training and Research Hospital, Clinic of Urology, Niğde, Turkiye

Phone: +90 5062317354 E-mail: drahmetcihan@gmail.com ORCID-ID: orcid.org/0000-0001-5586-8673

Received: 29.07 .2020

Accepted: 04.09.2020

Cite this article as: Cihan A, Cihan E, Çakmak B. Perceived Stress and Accompanying Low Urine pH Are in Relation to Bladder Pain Syndrome. J Urol Surg 2021;8(2):98-105.

${ }^{\circ}$ Copyright 2021 by the Association of Urological Surgery / Journal of Urological Surgery published by Galenos Publishing House. 
tubular dysfunction are known causes of excessive renal acid load (4). Metabolic acidosis and diet-dependent renal acid load were found to be associated with higher glucocorticoid levels (5). Although data on the association of stress with renal acid load is limited, glucocorticoids are known to be stress response hormones. Emotional stress is known to be a potential trigger of symptom exacerbations in patients with interstitial cystitis/BPS (6-8). At present, clinicians are quantifying the level of stress using valid instruments rating the perceived level of stress in each patient. The relationship between urine acidity level and perceived stress in patients with BPS has not been investigated, and whether perceived stress can be a trigger or mediator of acidic urine is still unclear. This issue provided the basis for our second hypothesis: Perceived stress accompanied by excessive urinary acid load may be associated with the presence and symptom severity of BPS. Therefore, this study aimed to evaluate the accompanying perceived stress levels of patients with BPS in comparison with healthy participants.

\section{Materials and Methods}

\section{Study Design and Setting}

A prospective case-control study was conducted to test the aforementioned hypotheses. The trial was started in August 2018 and ended in February 2020 after approval from review board (CU-06-07-2018/No: 45). During the recruitment period, potential participants who have given written consent for taking part in the study were evaluated by the researchers in the hospital setting. The patient group consisted of participants aged $>18$ years who were admitted to the urology clinic with complaints related to BPS and were newly diagnosed with BPS after all appropriate diagnostic tests and consultations were performed. As accepted currently, we used the definition "An unpleasant sensation (pain, pleasure, or discomfort) perceived to be related to the urinary bladder, associated with lower urinary tract symptoms of more than six weeks in the absence of other identifiable causes" to establish a diagnosis of BPS $(9,10)$. When patients presented with gross or microscopic haematuria, cystoscopy with hydrodistension under general anaesthesia was performed as needed. The control group consisted of volunteers aged $>18$ years who visited the gynaecology and/or urology outpatient clinic for reasons except as being a patient (relative of a patient, hospital staff, etc.) and who had no complaints related to cystitis or pelvic pain. Spot urine analysis was performed on the control group as a sole laboratory investigation.

Following the written informed consent process, all study participants were asked to rate their complaints using three questionnaires during the interview. In this study, translated forms of the O'Leary-Sant Interstitial Cystitis Symptom index (ICSI) (Appendix A) and O'Leary-Sant Interstitial Cystitis Problem index (ICPI) (Appendix B) were used to measure cystitis symptoms $(11,12)$. Since linguistically validated interstitial cystitis questionnaires were not available, both questionnaires were translated from their original English by an English language teaching professional for the current study. The validated version of the 10-item Perceived Stress scale (PSS-10) was used to measure the perceived stress levels of the participants in the last 4 weeks (13-15). Changes in PSS-10 scores ranged from 0 to 40 points, with higher scores indicating higher levels of perceived stress (13-15). Participants were asked to rate their pain levels using a 10-point (0-10) Likert visual analogue scale (VAS). Quality-of-life (0OL) was rated with a 6-point (0-5) Likert scale, with a higher score indicating better QOL. Patients with central or peripheral nervous system disease and taking antidepressants, antipsychotic, anxiolytic, anticholinergic, alpha blocker, mirabegron, or gabapentinoid medications were excluded from the study. Participants who rated either the ICSI or ICPI questionnaires above 6 points were excluded from the control group. The flowchart of the study is shown in Figure 1.

\section{Study Size and Data Handling}

Ninety-two participants for each group have been targeted according to sample size estimation using $\mathrm{G}^{*}$ Power software, with 70\% power, type 1 error of.05 and type 2 error of. 80 . Age, body mass index (BMI), current complaints, duration of complaints, comorbidities, medications and abnormal physical examination findings were recorded for each participant. Routine laboratory and radiologic examination findings were also recorded. The main variables investigated between the case and control groups included perceived stress levels (PSS-10) and spot urine $\mathrm{pH}$ as independent variables and O'Leary-Sant indexes (ICSI and ICPI), VAS-pain and QOL scores as dependent variables.

\section{Statistical Analysis}

At the beginning of the data analysis, Kolmogorov-Smirnov normality test was performed. The independent samples t-test was performed for all normally distributed variables during group comparisons. Non-parametric test was performed on variables that demonstrated a skewed distribution. The reliability of the linguistically translated ICSI and ICPI questionnaires were analysed using Cronbach's alpha test. A multivariate analysis was performed with logistic regression for nominal variables and linear regression for normally distributed dependent variables. In skewed distribution, logarithmic transformation was utilised before rendering the variables into a parametric regression. We used the Hosmer-Lemeshow test during the multivariate analysis with a binary logistic regression model for the final outcome. Hierarchical linear regression and path analysis for the measured dependent variables in the patient group were applied. While this study was planned in a prospective manner, 

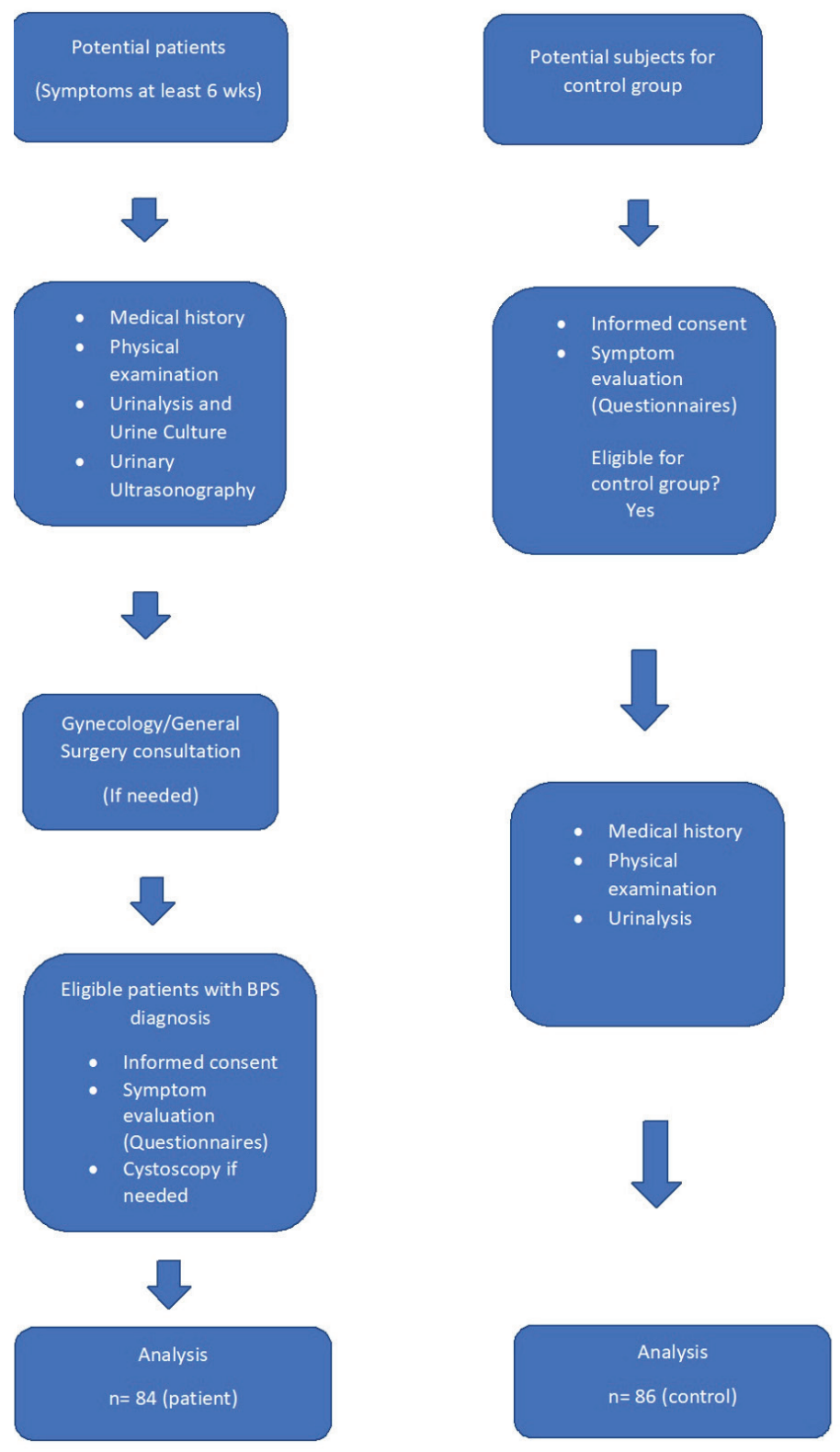

Figure 1. Flowchart of the study

none of the matching techniques were anticipated. All analyses were performed using the PASW Statistics (IBM, Armonk, NY, USA).

\section{Potential Biases}

Selection bias was the main confounder of this study. Thus, strict exclusion criteria and previously validated cut-off scores for ICSI and ICPI were used to deal with bias during the recruitment period.

\section{Results}

A total of 170 participants were recruited for the study. The patient group included 84 patients with BPS (73 women and 11 men), and 86 healthy participants (70 women and 16 men) were included in the control group. Only the BMI was significantly different between the patient and control groups. Demographic characteristics and comorbidities are shown in Table 1. Among patients with BPS, $42(50 \%)$ patients had pain in the pelvic region, $15(17.9 \%)$ in the pelvic and lumbar regions and $27(32.1 \%)$ in the pelvic and lumbar regions with involvement of other body sites. The mean pain duration was $23.6( \pm 30.7)$ months. The mean serum creatinine $(\mathrm{mg} / \mathrm{dL})$ and serum C-reactive protein values were $0.83( \pm 0.19)$ and 5.7 $( \pm 15.6)$, respectively. Accompanying nephrolithiasis (range, 3-8 mm) was found in $17(20.2 \%)$ patients. Cystoscopy was performed in $19(22.6 \%)$ patients. Ulcerative cystitis was found in $2(2.4 \%)$ patients, non-ulcerative cystitis with glomerulations in $13(15.5 \%)$ patients and normal bladder appearance in the remaining 4 (4.8\%) patients.

Among the study variables, only urine $\mathrm{pH}$ values demonstrated a skewed distribution in both groups (positive skewness of $0.59 \pm 0.26$ in the control group and $1.51 \pm 0.26$ in the BPS group). Therefore, we used the Mann-Whitney $U$ test to compare the urine $\mathrm{pH}$ values between the groups. The mean spot urine $\mathrm{pH}$, PSS-10, ICSI, ICPI, VAS-Pain and QOL scores were different between the patient and control groups. Results of comparative analysis within the groups are shown in Table 2. A reliability analysis of the items on the interstitial cystitis questionnaire revealed an intermediate level of internal consistency for the translated versions of the ICSI (Cronbach's alpha value of 0.78) and ICPI (Cronbach's alpha value of 0.84).

Initially, a binary logistic regression model was constructed for the presence of BPS. At the first block of the model, age and BMI were entered. The second block included spot urine $\mathrm{pH}$, spot urine density and PSS-10 scores. Spot urine $\mathrm{pH}$ levels $[p=0.01$, odds ratio $(O R)=0.31$ (95\% confidence interval (Cl) $0.12-0.75)]$ and PSS-10 scores $[p=0.01,0 R=1.1(95 \% \mathrm{Cl}$ 1.0-1.2)] were significant predictors in the final model. A hierarchical regression for ICSI, ICPI, VAS-Pain and QOL scores in the patient group revealed that only PSS-10 scores predicted the ICPI [standardised coefficient $\mathrm{B}=0.34(\mathrm{p}=0.01)$ ] and VASPain [standardised coefficient $B=0.35(p=0.01)$ ] scores and were independent from spot urine $\mathrm{pH}$, age and BMI. The path analysis with the study cohort also revealed significant correlations, as shown in Figure 2. Among the participants, ICSI, ICPI, VASPain and $\mathrm{OOL}$ scores were associated with both perceived stress levels and spot urinary $\mathrm{pH}$ levels. Lower urine $\mathrm{pH}$ and higher perceived stress were associated with worse ICSI, ICPI, VAS-Pain and $\mathrm{QOL}$ scores. The perceived stress scores were affected by ICPI and VAS-Pain levels. No predictor of urine $\mathrm{pH}$ levels was found among the study variables. 


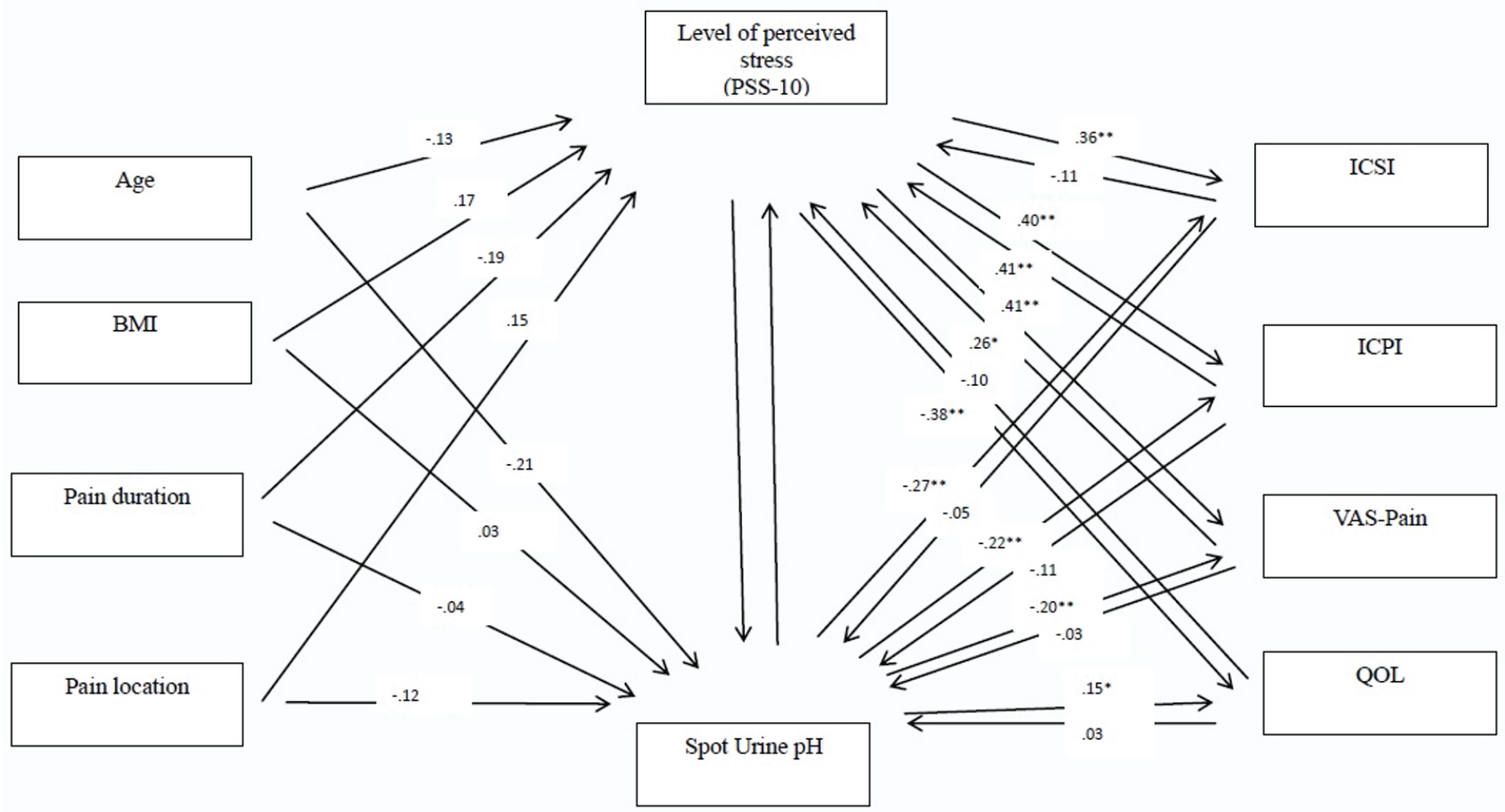

Figure 2. Results of the multivariable path analysis that demonstrates the relations of urine $\mathrm{pH}$, perceived stress and BPS symptoms. Correlation coefficients measured in the multivariable regression analysis are shown in the figure

*indicates $p \leq 0.05$ and **indicates $p<0.01$

Pain duration: Numerically coded as months

Pain location: Categorically ordered as 1 for pelvic region only, 2 for pelvic and lumbar region and 3 for pelvic plus lumbar region and other body parts, BPS: Bladder pain syndrome, BMI: Body mass index, VAS: Visual analogu scale, QOL: Quality-of-life

\begin{tabular}{|l|l|l|l|}
\hline \multicolumn{4}{|l|}{ Table 1. Characteristics of the study participants } \\
\hline & $\begin{array}{l}\text { Control } \\
\text { group } \\
\mathbf{n = 8 6}\end{array}$ & $\begin{array}{l}\text { Patient } \\
\text { group } \\
\text { (BPS) } \\
\mathbf{n = 8 4}\end{array}$ & p-value \\
\hline Age (years) & $39.7 \pm 10.4$ & $42.4 \pm 11.4$ & 0.11 \\
\hline Gender (F/M) & $70 / 16$ & $73 / 11$ & - \\
\hline BMI (kg/m²) & $25.8 \pm 4.6$ & $27.7 \pm 4.7$ & $0.01^{* *}$ \\
\hline Diabetes mellitus & $1(1.1 \%)$ & $5(5.9 \%)$ & 0.11 \\
\hline Hypertension & $7(6.0 \%)$ & $11(13 \%)$ & 0.32 \\
\hline Asthma ( \pm allergy) & $1(1.1 \%)$ & $5(5.9 \%)$ & 0.11 \\
\hline Thyroid dysfunction & $10(11.6 \%)$ & $7(8.3 \%)$ & 0.61 \\
\hline Other disease & $6(6.9 \%)$ & $14(16.6 \%)$ & 0.059 \\
\hline Smoking history (yes) & $20(23.2 \%)$ & $14(16.6 \%)$ & 0.24 \\
\hline Alcohol consumption (yes) & $2(1.7 \%)$ & $1(1.1 \%)$ & - \\
\hline $\begin{array}{l}\text { *significant at the level of 0.05 (with two-sided analysis) } \\
\text { **significant at the level of 0.01 (with two-sided analysis), BPS: Bladder pain syndrome, } \\
\text { BMl: Body mass index }\end{array}$ \\
\hline
\end{tabular}

\section{Discussion}

In this case-control study, we investigated the potential interrelations and predictor roles of urine $\mathrm{pH}$ and perceived stress level of patients with BPS. We found that low urine $\mathrm{pH}$

\begin{tabular}{|c|c|c|c|}
\hline & $\begin{array}{l}\text { Control group } \\
n=86\end{array}$ & $\begin{array}{l}\text { Patient group } \\
\text { (BPS) } \\
n=84\end{array}$ & p-value \\
\hline Spot urine $\mathrm{pH}$ & $6.2 \pm 0.5$ & $5.8 \pm 0.6$ & $0.0001^{* *}$ \\
\hline Spot urine density & $1.016 \pm 0.007$ & $1.015 \pm 0.007$ & 0.91 \\
\hline PSS-10 score & $18.3 \pm 5.7$ & $22.9 \pm 6.0$ & $0.0001^{* *}$ \\
\hline ICSI score & $3.2 \pm 1.5$ & $10.6 \pm 3.7$ & $0.0001^{* *}$ \\
\hline ICPI score & $3.1 \pm 1.8$ & $10.6 \pm 3.2$ & $0.0001^{* *}$ \\
\hline VAS-Pain score & $2.0 \pm 1.7$ & $6.0 \pm 2.5$ & $0.0001^{* *}$ \\
\hline QOL score & $2.7 \pm 1.4$ & $1.4 \pm 1.1$ & $0.0001^{* *}$ \\
\hline \multicolumn{4}{|c|}{$\begin{array}{l}\text { *significant at the level of } 0.05 \text { (with two-sided analysis) } \\
\text { *significant at the level of } 0.01 \text { (with two-sided analysis), BPS: Bladder pain syndrome, } \\
\text { PSS-10: The 10-item Perceived Stress scale, ICSI: Interstitial Cystitis Symptom index, } \\
\text { ICPI: Interstitial Cystitis Problem index, VAS-Pain: Visual analogue scale for pain, QOL: } \\
\text { Quality-of-life }\end{array}$} \\
\hline
\end{tabular}

and increased perceived stress predicted the presence of BPS. The urine $\mathrm{pH}$ and perceived stress levels correlated with ICSI, ICPI, VAS-pain and QOL scores independent from each other.

The ICSI, ICPI, VAS-Pain and OOL scores were significantly different between the groups. Although linguistically validated ICSI and ICPI questionnaires are still not available in our country, 
the clinical validity of ICSI and ICPI for patients with BPS has already been confirmed $(11,12)$. O'Leary et al. (11) concluded that nearly all patients with BPS have given both ICSI and ICPI questionnaires ratings higher than 6 points. For this reason, we used a cut-off of 6 points while rendering participant to our control group to avoid selection bias. As the ICSI and ICPI questionnaire results showed acceptable internal consistency, we believed that our study groups were constructed appropriately.

We performed cystoscopy with hydrodistension under general anaesthesia as needed. Although recent trials have recommended routine cystoscopic phenotyping of patients with BPS during the initial diagnostic evaluation, our cystoscopic findings, in terms of ulcerative cystitis prevalence, or normal findings do not deviate from those in the literature (16-18). Additionally, we found relatively high rates of nephrolithiasis in our patients with BPS compared with the current known prevalence (19). To the best of our knowledge, no data were available on the prevalence rate of nephrolithiasis in patients with BPS. Further clinical studies are needed on this topic, while we can only speculate that acidic urine $\mathrm{pH}$ may increase the risk of kidney stone formation in patients with BPS.

As a novel finding of this study, an increase in the level of urine $\mathrm{pH}$ is associated with lower odds for the presence of BPS. Our path analysis also revealed that acidic spot urine $\mathrm{pH}$ levels predicted worse symptom scores in the patients. Moreover, the urine $\mathrm{pH}$ level is not correlated with any patient characteristics or perceived patient stress. Nguan et al. (20) reported the first investigation of the effect of urine $\mathrm{pH}$ changes on pain scores of patients with interstitial cystitis in a double-blind crossover study. They found no difference in the pain scores of patients with intravesical instillation of either an acidic solution (pH 5.0) or a neutral buffered solution (pH 7.5) (20). In 2014, Ueda et al. (3) reported a significant improvement in micturition complaints and ICPI scores of patients with hypersensitive bladder by using urinary alkalisation therapy to increase the urine $\mathrm{pH}$ to 6.2. Recently, Sönmez et al. (2) also demonstrated that urine alkalisation therapy improves voiding symptoms and pain scores of women with acidic urine $(\mathrm{pH}<6)$ after 4 weeks. Although no physiopathological data exist on the relationship between urine $\mathrm{pH}$ level and BPS symptoms, existing preclinical data speculate that the expression of TRPV1 capsaicin and acid-sensing ion channel receptors at the terminals of bladder afferent $C$ fibre terminals might be responsible for symptom improvement through urine alkalisation in women diagnosed with hypersensitive bladder and those with acidic urine $(2,3,21-25)$. Thus, our results may promote current knowledge and might provide a clinical framework for further clinical and preclinical studies to clarify the relationship between acidic urine $\mathrm{pH}$ and BPS symptoms.

The higher level of perceived stress in the current study population indicates higher odds for the presence of BPS. In this study, an increase in perceived stress has direct disruptive effects on patients' complaints. The second hypothesis of this study was partially rejected because perceived stress was not associated with urine $\mathrm{pH}$ level. However, preclinical studies have demonstrated a relationship between water avoidance stress and severity of cystitis $(8,26)$. Previous clinical studies have also revealed that daily life stress and acute stress are related to increased urgency and pain ratings in patients with interstitial cystitis $(6,7,27)$. Autonomic nervous system hyperactivity and stress-induced hyperalgesia were initially the focus of trials investigating the pathogenetic relationship between stress and interstitial cystitis/BPS (28). Recently, Jhang et al. (29) demonstrated that the dysregulation of bladder corticotrophin-releasing hormone receptor subtypes has improved the pathogenetic theories on stress-induced symptom exacerbations in patients with BPS. In the present study, we measured the perceived stress level of the participants using a valid instrument and demonstrated that, in addition to the predictive value for BPS, the perceived stress of the participants was also influenced by their perception of the cystitis problem and pain level. A previous factor analysis of the PSS-10 revealed that the questionnaire constituted mainly of "inadequate self-efficacy" and "perception of stress discomfort" factors (13). A positive feedback mechanism might underlie the current correlations, i.e. "patients with BPS who have increasing pain and feel that they have problematic bladder complaints are also exposed to higher perceived stress level".

\section{Study Limitations}

First, the ICSI and ICPI questionnaires that were used were not linguisticaly validated. Second, we did not perform cystoscopy on all patients during the diagnostic evaluation of the case group.

\section{Conclusion}

Acidic urine $\mathrm{pH}$ and higher perceived stress levels are associated with the presence of BPS. Perceived stress independent from urine $\mathrm{pH}$ is related to BPS symptoms in a bidirectional manner. Physicians should take spot urine $\mathrm{pH}$ and perceived stress levels into account during decision making while evaluating patients with BPS. Furthermore, prospective placebo-controlled trials are needed to determine the therapeutic relevance of urinary $\mathrm{pH}$ and stress modifiers.

Acknowledgements: We wish to thank Ms. Betül Karamış, (Ph.D) for her support during translation of ICSI and ICPI questionnaires.

\section{Ethics}

Ethics Committee Approval: All study participants provided informed consent, and the study design was approved by the appropriate ethics review board (Çukurova University School of Medicine-Adana-CU-06-07-2018/no: 45). 
Informed Consent: During the recruitment period, potential participants who have given written consent for taking part in the study were evaluated by the researchers in the hospital setting.

Peer-review: Externally peer-reviewed.

\section{Authorship Contributions}

Surgical and Medical Practices: A.C., E.C., Concept: A.C., E.C., Design: A.C., E.C., Data Collection or Processing: A.C., E.C., Analysis or Interpretation: A.C., Literature Search: A.C., B.Ç., Writing: A.C., E.C., B.Ç.

Conflict of Interest: No conflict of interest was declared by the authors.

Financial Disclosure: The authors declare that they have no relevant financial.

\section{References}

1. Hanno P, Nordling J, Fall M. Bladder pain syndrome. Med Clin North Am 2011:95:55-73.

2. Sönmez MG, Göğer YE, Ecer G, Atıcı A, Özkent MS, Öztürk A. Effects of urine alkalinization with sodium bicarbonate orally on lower urinary tract symptoms in female patients: a pilot study. Int Urogynecol J 2018;29:10291033.

3. Ueda $T_{\text {, Yoshida }}$, Tanoue $H_{\text {, Ito }}$, Tamaki $M$, Ito $Y$, Yoshimura N. Urine alkalization improves the problems of pain and sleep in hypersensitive bladder syndrome. Int J Urol 2014;21:512-517.

4. Remer T. Influence of nutrition on acid-base balance--metabolic aspects. Eur J Nutr 2001;40:214-220.

5. Esche J, Shi L, Sánchez-Guijo A, Hartmann MF, Wudy SA, Remer T. Higher diet-dependent renal acid load associates with higher glucocorticoid secretion and potentially bioactive free glucocorticoids in healthy children. Kidney Int 2016;90:325-333.

6. Rothrock NE, Lutgendorf SK, Kreder KJ, Ratliff T, Zimmerman B. Stress and symptoms in patients with interstitial cystitis: a life stress model. Urology 2001;57:422-427.

7. Lutgendorf SK, Kreder KJ, Rothrock NE, Ratliff TL, Zimmerman B. A laboratory stress model for examining stress and symptomatology in interstitial cystitis patients. Urology 2001;57(6 Suppl 1):122

8. Hanna-Mitchell AT, Wolf-Johnston A, Roppolo JR, Buffington TC, Birder LA. Corticotropin-releasing factor family peptide signaling in feline bladder urothelial cells. J Endocrinol 2014;222:113-121.

9. Hanno PM, Erickson D, Moldwin R, Faraday MM; American Urological Association. Diagnosis and treatment of interstitial cystitis/bladder pain syndrome: AUA guideline amendment. J Urol 2015;193:1545-1553.

10. Doggweiler $R$, Whitmore KE, Meijlink JM, Drake MJ, Frawley $H$, Nordling J, Hanno P, Fraser MO, Homma Y, Garrido G, Gomes MJ, Elneil S, van de Merwe JP, Lin ATL, Tomoe H. A standard for terminology in chronic pelvic pain syndromes: A report from the chronic pelvic pain working group of the international continence society. Neurourol Urodyn 2017;36:984-1008.

11. O'Leary MP, Sant GR, Fowler FJ Jr, Whitmore KE, Spolarich-Kroll J. The interstitial cystitis symptom index and problem index. Urology 1997;49(5A Suppl):58-63.
12. Lubeck DP, Whitmore K, Sant GR, Alvarez-Horine S, Lai C. Psychometric validation of the $0^{\prime}$ leary-Sant interstitial cystitis symptom index in a clinical trial of pentosan polysulfate sodium. Urology 2001;57(6 Suppl 1):62-66.

13. Eskin $M$, Harlak $H$, Demirkıran $F$, Dereboy Ç. Algılanan Stres Ölçeğinin Türkçeye Uyarlanması: Güvenirlik ve Geçerlik Analizi. Yeni Symp J 2013;51:132-140

14. Cohen $\mathrm{S}$, Kamarck T, Mermelstein R. A global measure of perceived stress. J Health Soc Behav 1983;24:385-396.

15. Kaya C, Tansey TN, Melekoglu M, Cakiroglu O, Chan F. Psychometric evaluation of Turkish version of the Perceived Stress Scale with Turkish college students. J Ment Health 2019;28:161-167.

16. Whitmore $K E$, Fall $M$, Sengiku A, Tomoe $H$, Logadottir $Y$, Kim $Y H$. Hunner lesion versus non-Hunner lesion interstitial cystitis/bladder pain syndrome. Int J Urol 2019;26 Suppl 1:26-34.

17. Akiyama $Y$, Hanno P. Phenotyping of interstitial cystitis/bladder pain syndrome. Int J Urol. 2019;26 Suppl 1:17-19.

18. Acar Ö, Tarcan T. Cystoscopic evaluation and clinical phenotyping in interstitial cystitis/bladder pain syndrome. J Turk Ger Gynecol Assoc 2019;20:117-122.

19. Sorokin I, Mamoulakis C, Miyazawa K, Rodgers A, Talati J, Lotan Y. Epidemiology of stone disease across the world. World J Urol 2017;35:13011320.

20. Nguan C, Franciosi LG, Butterfield NN, Macleod BA, Jens M, Fenster HN. A prospective, double-blind, randomized cross-over study evaluating changes in urinary $\mathrm{pH}$ for relieving the symptoms of interstitial cystitis. BJU Int 2005;95:91-94.

21. Sánchez-Freire V, Blanchard MG, Burkhard FC, Kessler TM, Kellenberger $S$, Monastyrskaya K. Acid-sensing channels in human bladder: expression function and alterations during bladder pain syndrome. J Urol 2011:186:1509-1516.

22. Holzer P, Izzo AA. The pharmacology of TRP channels. Br J Pharmacol 2014;171:2469-2473.

23. Caterina MJ, Schumacher MA, Tominaga M, Rosen TA, Levine JD, Julius D. The capsaicin receptor: a heat-activated ion channel in the pain pathway. Nature 1997;389:816-824.

24. Tominaga $M$, Caterina MJ, Malmberg AB, Rosen $T A$, Gilbert $H$, Skinner $K_{\text {, }}$ Raumann BE, Basbaum Al, Julius D. The cloned capsaicin receptor integrates multiple pain-producing stimuli. Neuron 1998;21:531-543.

25. Jordt $S E$, Tominaga $M$, Julius D. Acid potentiation of the capsaicin receptor determined by a key extracellular site. Proc Natl Acad Sci U S A 2000;97:8134-8139.

26. Matos R, Serrão $P$, Rodriguez L, Birder LA, Cruz F, Charrua A. The water avoidance stress induces bladder pain due to a prolonged alpha $1 \mathrm{~A}$ adrenoceptor stimulation. Naunyn Schmiedebergs Arch Pharmacol 2017;390:839-844.

27. Lutgendorf SK, Kreder KJ, Rothrock NE, Ratliff TL, Zimmerman B. Stress and symptomatology in patients with interstitial cystitis: a laboratory stress model. J Urol 2000;164:1265-1269.

28. Mullins C, Bavendam T, Kirkali Z, Kusek JW. Novel research approaches for interstitial cystitis/bladder pain syndrome: thinking beyond the bladder. Transl Androl Urol 2015;4:524-533.

29. Jhang JF, Birder LA, Jiang YH, Hsu YH, Ho HC, Kuo HC. Dysregulation of bladder corticotropin-releasing hormone receptor in the pathogenesis of human interstitial cystitis/bladder pain syndrome. Sci Rep 2019;9:19169. 


\section{Appendix A}

Interstitial Cystitis Symptom Index (ICSI)

During the past month:

How often have you felt the strong need to urinate with little or no warning?

0 . Not at all

1. Less than 1 time in 5

2. Less than half the time

3. About half the time

4. More than half the time

5. Almost always

Have you had to urinate less than 2 hours after you finished urinating?

0 . Not at all

1. Less than 1 time in 5

2. Less than half the time

3. About half the time

4. More than half the time

5. Almost always

How often did you most typically get up at night to urinate?

0. None

1. Once

2. 2 times

3. 3 times

4. 4 times

5. 5 or more times

Have you experienced pain or burning in your bladder?

0 . Not at all

1. A few times

2. Almost always

3. Fairly often

4. Usually

Add the numerical values of the checked entries.

Total score 


\section{Appendix B}

Interstitial Cystitis Problem Index (ICPI)

During the past month, how much has each of the following been a problem for you:

Frequent urination during the day?

0 . No problem

1. Very small problem

2. Small problem

3. Medium problem

4. Big problem

\section{Getting up at night to urinate?}

0 . No problem

1. Very small problem

2. Small problem

3. Medium problem

4. Big problem

Need to urinate with little warning?

0 . No problem

1. Very small problem

2. Small problem

3. Medium problem

4. Big problem

Burning, pain, discomfort, or pressure in your bladder?

0 . No problem

1. Very small problem

2. Small problem

3. Medium problem

4. Big problem

Add the numerical values of the checked entries.

Total score 